\title{
Polarization and angular peculiarities of IR emission of thin film semiconductor structures
}

\author{
O.G. Kollyukh, A.I. Liptuga, V.O. Morozhenko, V.I. Pipa \\ Institute of Semiconductor Physics, NAS of Ukraine, 45 prospect Nauky, 03028 Kyiv, Ukraine \\ Phonelfax: +38(044) 265 6361, e-mail: kollyukh@isp.kiev.ua
}

\begin{abstract}
In this paper thermal radiation from plane-parallel semiconductor layers was investigated. Shown is that spectra of thermal radiation from structures has an oscillating character caused by multi-beam interference. It was shown that the density of thermal radiation, at its interference maximum, can be equal to half the density of thermal radiation from a blackbody source, at the same time at the interference minimum the value approached practically zero. In addition, the angular dependence of thermal radiation does not obey the Lambert law and demonstrates a non-monotonic character with clearly pronounced extrema.
\end{abstract}

Keywords: thermal radiation, interference, polarization.

Paper received 28.01.03; accepted for publication 16.06.03.

If the temperature of a body rises above absolute zero, the body emits light. The characteristics of thermal radiation depend on the optical and geometrical parameters of the emitting body as well as its temperature $T$. The spectral intensity of the emission $J(\omega, T)$ from the surface is determined in accordance with Kirchhoff's law by the expression:

$$
J(\omega, T)=A\left(\omega, \vartheta_{1}\right) U(\omega, T) \cos \theta_{1},
$$

where $\omega$ is the frequency, $A\left(\omega, \vartheta_{1}\right)$ is directional spectral absorbtivity (emissivity), $\theta_{1}$ is the angle between the normal of the emitting surface and the direction of observation. $U(\omega, T)$ - density of the energy flux of black body radiation which is determined by Planck's formula:

$$
U(\omega, T)=\frac{\hbar \omega^{3}}{4 \pi^{3} c^{2}\left(e^{\hbar \omega / k_{B} T}-1\right)},
$$

where $k_{B}$ is Boltzmann's constant. The coefficient $A\left(\omega, \vartheta_{1}\right)$ determines the fraction of the energy flux of external equilibrium radiation which is absorbed by the body. For an absolute black body, $A\left(\omega, \vartheta_{1}\right)=1$. In expression (1) the same coefficient determines which frac- tion of black body radiation is emitted by a real body. Below, when analyzing thermal radiation, we will refer to coefficient $A\left(\omega, \vartheta_{1}\right)$ as emissivity.

Let us consider, for example, a layer with thickness $l$, coefficient of absorption $\alpha(\omega, T)$ and coefficient of reflection, from one surface only, $R\left(\omega, \vartheta_{1}\right)$. When the layer is opaque $(\alpha(\omega, T) l>>1)$, and the angle of total internal reflection is small, then $A=1-R(\omega)$, where $R(\omega)$ is the reflection coefficient for normal incidence. For a transparent layer $(\alpha(\omega, T) l<<1), A=\alpha(\omega, T) l[1-3]$. These results could lead one to believe that these dependencies describe completely all the peculiarities of thermal radiation. Nevertheless, there are certain systems, thermal emission of which deviates dramatically from that described in [1-3]. The aim of this paper is to study the thermal emission of semitransparent plane parallel structures (such as Fabry-Perot resonator). The peculiarities of thermal radiation of thin layers were first predicted in the quantum theory [4]. We show that peculiarities of thermal radiation, which we measured in our systems, are well described by Kirchhoff's law (1), if classic multibeam interference is taken into account in $A\left(\omega, \vartheta_{1}\right)$.

Let us consider a plane parallel layer $(0 \leq z \leq l)$ placed on a substrate $(z>l)$, the surface $z=0$ being in a contact with vacuum. We will refer to these media 


\section{O.G. Kollyukh et al.: Polarization and angular peculiarities of IR emission ...}

(vacuum, layer and substrate) as $j=1,2,3$, respectively. For a plane wave incident onto the surface between the adjacent $j$ and $i$ media, the Fresnel reflection amplitudes are given by

$$
r_{i j}^{(s)}=\frac{k_{i z}-k_{j z}}{k_{i z}+k_{j z}}, r_{i j}^{(p)}=\frac{n_{j}^{2} k_{i z}-n_{i}^{2} k_{j z}}{n_{j}^{2} k_{i z}+n_{i}^{2} k_{j z}},
$$

where the labels $s, p$ refer to the reflection amplitudes of waves which have $s$ - and $p$-polarization. In Eq. (3),

$$
k_{j z}=\sqrt{\frac{\omega^{2}}{c^{2}} n_{j}^{2}-k_{\tau}^{2}},
$$

$n_{j}$ is the complex refractive index, $k_{\tau}$ is the in-plane wave vector: $\vec{k}_{j}=\left(\vec{k}_{\tau}, k_{j z}\right)$. Note, that in the case of normal incidence $\left(k_{\tau}=0\right), r_{i j}^{(p)}=-r_{i j}^{(s)}$.

Consider a linear polarized plane wave incident upon the layer from the vacuum with the angle of incidence $\theta_{1}$. In this case, $k_{\tau}=(\omega / c) \sin \theta_{1}$. The amplitudes of reflection from the layer are given by

$$
r_{s}=\frac{r_{12}^{(s)}+r_{23}^{(s)} e^{2 i k_{2 z} l}}{1+r_{12}^{(s)} r_{23}^{(s)} e^{2 i k_{2 z} l}}, r_{p}=\frac{r_{12}^{(p)}+r_{23}^{(p)} e^{2 i k_{2 z} l}}{1+r_{12}^{(p)} r_{23}^{(p)} e^{2 i k_{2 z} l}} .
$$

The amplitudes of transmission are

$$
\begin{aligned}
& t_{s}=\frac{\left(1+r_{12}^{(s)}\right)\left(1+r_{23}^{(s)}\right)}{1+r_{12}^{(s)} r_{23}^{(s)} e^{2 i k_{2 z} l} e^{i k_{2 z} l},} \\
& t_{p}=\left(\frac{n_{1}}{n_{3}}\right) \frac{\left(1+r_{12}^{(p)}\right)\left(1+r_{23}^{(p)}\right)}{1+r_{12}^{(p)} r_{23}^{(p)} e^{2 i k_{2 z} l}} e^{i k_{2 z} l} .
\end{aligned}
$$

Using Eqs (5) and (6), we may write the absorptivity of the structure under consideration as follows

$$
A_{v}=1-\left|r_{v}\right|^{2}-\frac{k_{3 z}}{k_{1 z}}\left|t_{v}\right|^{2}
$$

where $v=s, p$. Below, we assume that $\operatorname{Im} n<<\operatorname{Re} n$. Under this assumption, the reflection factors $r_{i j}$ may be treated as real values, absorption of radiation is taken into account only in the exponential factors in Eqs (5) and (6). While passing through the layer the decrease of intensity is given by the factor $\eta=\exp \left(-\alpha l / \cos \theta_{2}\right)$, where the angle $\theta_{2}$ is related to the angle of incidence by the relationship $n_{1} \sin \theta_{1}=n_{2} \sin \theta_{2}$. Finally, for absorptivity $A_{v}$ we get

$$
A_{v}=\frac{\left(1-r_{12}^{2}\right)\left(1+\eta r_{23}^{2}\right)(1-\eta)}{1+2 r_{12} r_{23} \cos \left(2 k_{2 z} l\right) \eta+r_{12}^{2} r_{23}^{2} \eta^{2}},
$$

where $r_{i j}=r_{i j}(s, p)$ is used for $A_{s, p}$. When the incident light is totally non-polarized, the absorptivity is determined as $A=\left(A_{s}+A_{p}\right) / 2$.

$S Q O, 6(2), 2003$
If in Eq. (8) $2 k_{2 z} l \equiv \phi>>1$, the absorptivity $A_{v}(\omega)$ is a fast oscillating function of $\omega$. Averaging $A_{v}(\omega)$ over the phase $\phi$ (the situation which is realized for a nonplaneparallel structure) gives the expression

$\bar{A}_{V}=\frac{\left(1-R_{12}\right)\left(1+\eta R_{23}\right)(1-\eta)}{1-R_{12} R_{23} \eta^{2}}$,

where $R_{i j}=r_{i j}^{2}$. For the optically thin layer (either because of weak absorption or small thickness) which is placed in vacuum $\left(R_{12}=R_{23}=R\right)$, and for radiation propagating close to the normal, absorptivity (9) is reduced to $\bar{A}_{v} \approx \alpha(\omega, T) l$.

If the plate is partially transparent and strictly plane parallel, then general expression (8) for the absorptivity, which takes into consideration interference, should be used in calculations of thermal emission. Thus, in order to calculate the spectral intensity of thermal emission and its polarization properties, we will use the expression

$$
J_{v}\left(\omega, \theta_{1}\right)=A_{v}\left(\omega, \theta_{1}\right) U(\omega) \cos \theta_{1},
$$

where emissivity $A_{v}\left(\omega, \vartheta_{1}\right)$ is given by Eq. (8). From Eqs. (8) and (10), it follows that the spectral intensity of thermal emission of a plane-parallel plate oscillates as a function of the frequency and of the angle of observation. The amplitude modulation depends on polarization of emitted radiation.

The spectral emissivity calculated from Eqs (8) and (9) as a function of a wavelength $\lambda$ is shown in Fig. 1 . The calculation was carried out for $\theta_{1}=0$ using the following parameters: $l=40 \mu \mathrm{m}, n=3.4, R=0.3, \alpha=110 \mathrm{~cm}^{-1}$ (we assumed that $n, R, \alpha$ do not depend on $\lambda$ ). As seen from Fig. 1, the emissivity of a plane-parallel plate (curve 1) ranges from 0.21 to 0.46 . At the same time, the emissivity of a non-plane-parallel plate that has the same parameters, does not depend on $\lambda$ and equals to 0.31 .

The similar behavior of the emissivity was measured experimentally (Fig. 2). Emissivity of a plane-parallel plate of $n$-GaAs ( curve 1 ) oscillates around the emissivity of the non plane-parallel plate (curve 2). The non plane-parallel plate was made of the same material with a small angle between its faces (about 0.1 degree), its mean thickness was equal to $l$.

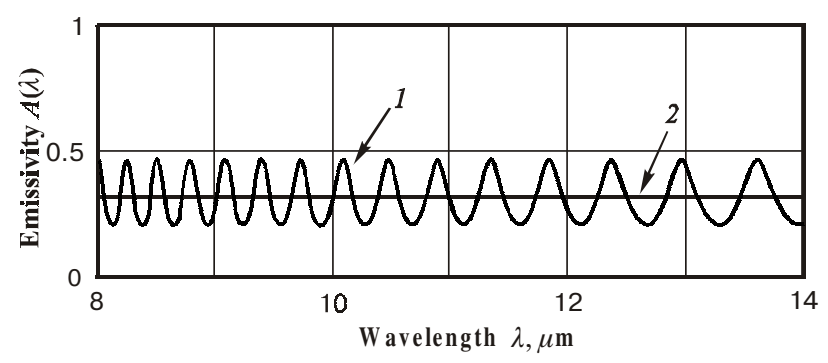

Fig. 1. Spectral emissivity of plane-parallel (1) and nonplaneparallel (2) plates. 


\section{O.G. Kollyukh et al.: Polarization and angular peculiarities of IR emission ...}

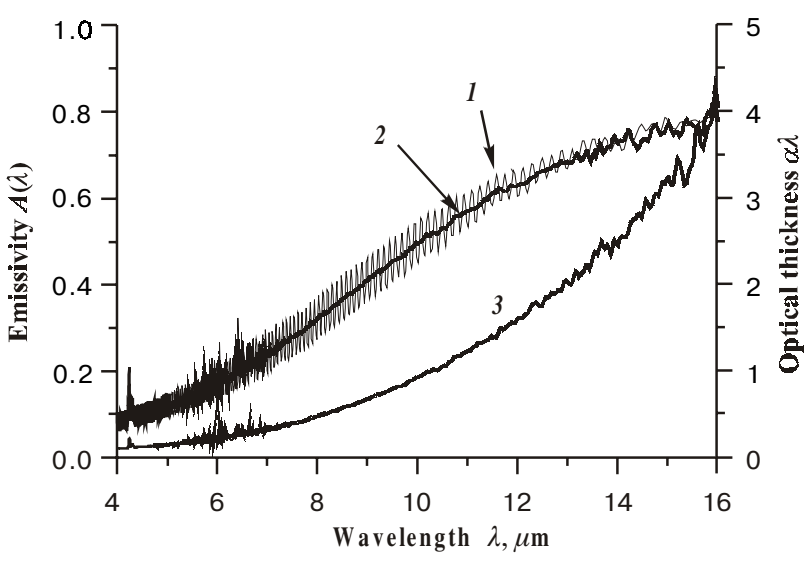

Fig. 2. Experimental spectral emissivity, $A(\lambda)$ for plane-parallel (curve 1) and nonplane-parallel (curve 2) plates of $n$-GaAs. Curve 3 represents the dependence of the optical thickness of the plates, $\alpha l$, on $\lambda$. The plate thickness is $l=110 \mu \mathrm{m}$, the concentration of noncompensated impurities is $N_{d}-N_{a}=1 \cdot 10^{18} \mathrm{~cm}^{-3}, T=$ $=360 \mathrm{~K}$.

The amplitude of the oscillations $\left(\Delta A(\lambda)=A_{\max }-\right.$ $\left.-A_{\min }\right)$ depends on $\lambda$ non-monotonically. With increasing $\lambda$, the amplitude $\Delta A(\lambda)$ gradually increases, passes over maximum (at $\lambda \approx 8.3 \mu \mathrm{m}$ ), then decreases and drops practically to zero at $\lambda>12 \mu \mathrm{m}$ due to strong absorption (the optical thickness $\alpha l>1.6$ ). The spectral position of the maximal amplitude $\left(\Delta A_{\max }\right)$ is determined by the values of the optical parameters values $R$ and $\alpha l$.

If $R$ value is known and frequency dispersion of $n$ is weak, the spectral position of $\Delta A_{\max }$ makes it possible to determine $\alpha l$ magnitude (or vice versa, to determine $R$ value if $\alpha l$ is known). However, an attempt to determine $R$ from the data presented in Fig. 2 has resulted in overstated value of $\alpha l$. This is explained by the fact that the material used has a noticeable dispersion of the refractive index. The dependence $n(\lambda)$ removes $\Delta A_{\max }$ to the short-wavelength range. For the same reason, in the spectral range $12-14 \mu \mathrm{m}$, where the sample is opaque (interference is not recorded), the emissivity increases [5].

Our analysis shows that the maximal swing of emission, i.e. the difference between the intensity that corresponds to the nearest neighbor maximum and minimum, is realized when $R \rightarrow 1$ and $\alpha(\omega, T) l \rightarrow 0$. This makes it possible to create effective thermal IR-sources that have a modulated intensity of radiation. The use of semiconductor materials, where the condition $R \rightarrow 1$ can be provided in a relatively simple way using a dielectric coating, looks promising.

The study of angle and polarization dependencies of thermal radiation were carried out for $\mathrm{Si}$ samples. The emissivity of the plane-parallel sample, $A\left(\vartheta_{1}\right)$, normalized at the emissivity $\bar{A}$ of the nonplane-parallel sample, which has analogous parameters, is shown in Fig. 3. As can be seen, the angle dependencies of the normalized emissivity, $A^{*}\left(\theta_{1}\right)=A / \bar{A}$, for unpolarized radiation (Fig. $3 a)$ and radiation which is polarized in the plane of incidence ( $s$ - polarization, Fig. 3 b) are qualitatively similar
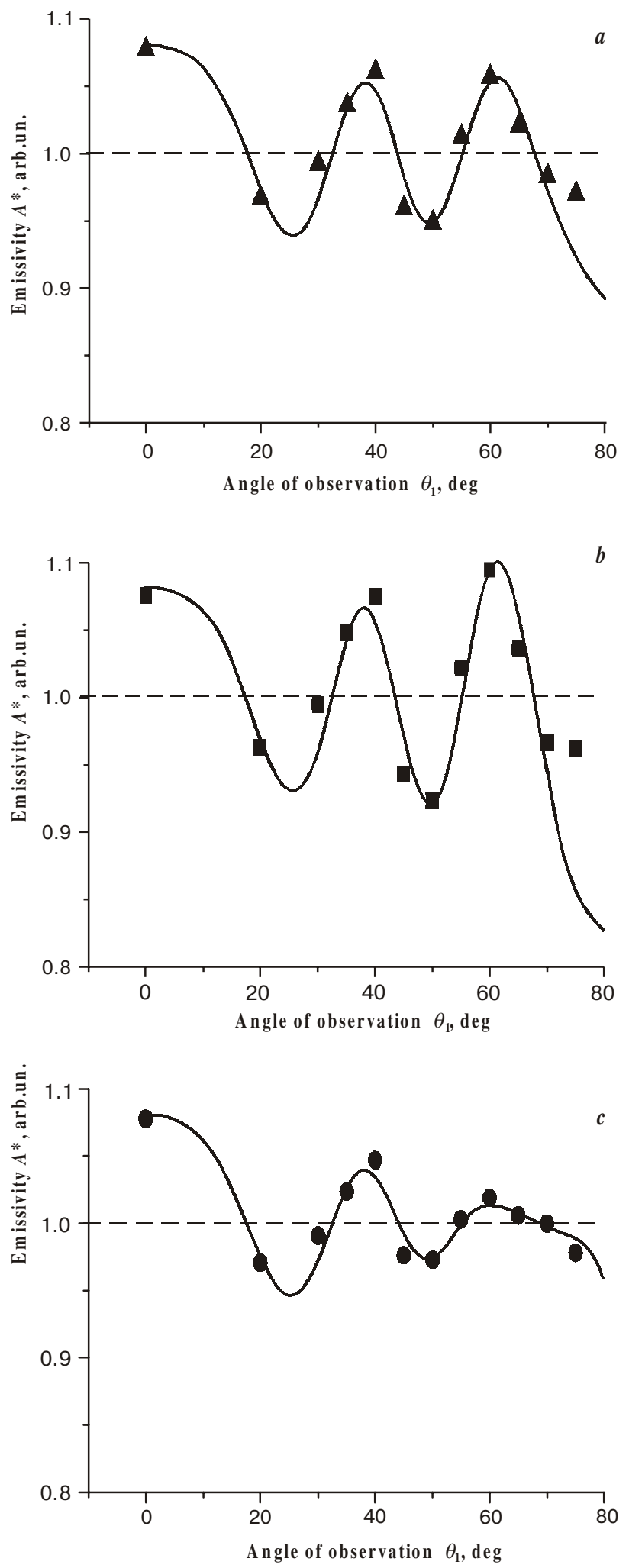

Fig. 3. Dependencies of normalized emissivity $A^{*}\left(\theta_{1}\right)$ of plane parallel plate of Si on the angle of observation $\theta_{1} ; \lambda=10.57 \mu \mathrm{m}$, $l \approx 90 \mu \mathrm{m}, T=360 \mathrm{~K}, a-$ unpolarized radiation, $b-s$-polarization, $c-p$-polarization. The solid lines show the theoretical results, the dotted lines show the experimental data. 


\section{O.G. Kollyukh et al.: Polarization and angular peculiarities of IR emission ...}

to each other. They have non-monotonic character with clearly noticeable extrema. For the $s$ - polarized radiation, the magnitudes of the extrema increase as the angle of observation increases. For radiation which is polarized in the plane of incidence ( $p$-polarization, Fig. $3 c)$, the dependence $A^{*}\left(\theta_{1}\right)$ differs qualitatively from the dependencies presented in Figs $3 \mathrm{a}, 3 \mathrm{~b}$. In the angle range $55^{\circ}-75^{\circ}$, extrema practically vanish, which indicates a violation of the resonance due to decreasing the reflection coefficient almost to zero.

The difference between the angle distribution of radiation which is emitted by plane-parallel and nonplaneparallel plates is well seen from Fig. 4. In this figure the calculated angle dependence of the spectral intensities of unpolarized thermal radiation, the function $A\left(\vartheta_{1}\right) \cos \vartheta_{1}$, is presented by curve 1 for a plane-parallel plate and by curve 2 for a non-plane-parallel plate. The calculations were carried out for silica plate with the thickness $l=90 \mu \mathrm{m}$. The dependence presented by curve 2 (in this case, $\left.A\left(\vartheta_{1}\right)=\bar{A}\left(\vartheta_{1}\right)\right)$ is described by a law close to Lambert's law [6], the possible deviations from this law at large angles are caused by the angle dependence of the reflection coefficient $R\left(\vartheta_{1}\right)$. On comparing curves 1 and 2 , one can see that multi-beam interference of thermal radiation exerts a sufficient influence on the angular distribution of radiation. For a semi-transparent plane-parallel plate, the angular distribution of thermal radiation

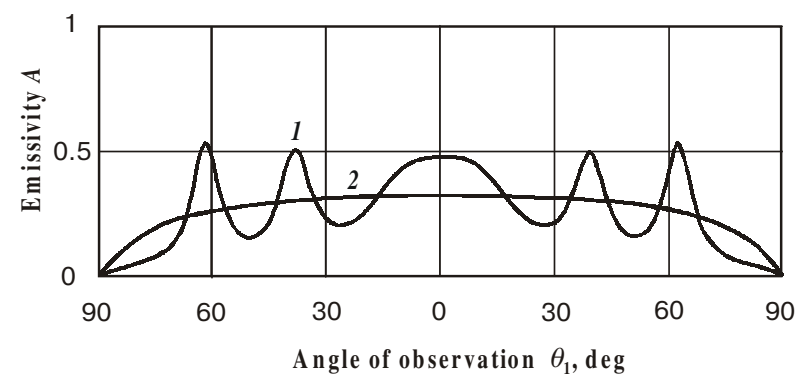

Fig. 4. Theoretical angular dependencies of unpolarized radiation from plane-parallel (curve 1) and non-plane-parallel (curve 2) plates of $\mathrm{Si}, l=90 \mu \mathrm{m}$. does not obey Lambert's law. This theoretical result is well confirmed by our experimental measurements.

Thus, the thermal radiation spectrum of a plane-parallel plate differs significantly from the thermal radiation spectrum of an identical non-plane-parallel plate. Due to multi-beam interference, a smooth spectral dependence of radiation is transformed into an oscillating dependence with sharp peaks. In addition, the angular dependence of thermal radiation does not obey Lambert's law and demonstrates a non-monotonic character with clearly manifested extrema. The density of thermal radiation, at its interference maximum, can be equal to half the density of thermal radiation from a black body source, at the same time at the interference minima the value approached practically zero. These limiting characteristics of radiation are realized if $R \rightarrow 1$. This makes it possible to create effective thermal IR-sources, operating spectral band is given by the emission element parameters. Such sources can work in different regions of IR-spectrum. Best of all is to use semiconductor plates, because of their optical parameters, and hence the density of radiation can be modulated by various known modes.

\section{References}

1. Moss T.S., Burrel G.J., Ellis B., Semiconductor Opto-Electronics, Butterworths, London, (1973).

2. B.I.Stepanov, Ya.S.Hvashchevskaya, Determination of absorption coefficient from the spectra of thermal emission of translucent plane-parallel layers // Inzhenerno-fizicheskiy Zhurnal, 1(10), pp. 82-87 (1958).

3. V.K.Malyutenko, A.I.Liptuga, G.I.Teslenko and V.A.Botte Thermal emission of semiconductors under nonequilibrium conditions // Infrared Phys. 29 pp.693-700 (1989).

4. V.S.Pekar Peculiarities of optical spectra of thin layers // Journal of Experimental and Theoretical Phys. 88, pp.667676 (1985)

5 V.K.Malyutenko, V.A.Morozhenko, V.I.Chernyakhovsky Influence of plasma oscillations on thermal emission spectrum of doped semiconductors // Infrared Phys. 33, pp.583587 (1992).

6. G.Gaussorgurs, La Thermographie Infrarouge, PrincipesTechnologie-Applications. In: Technique et Documentation, Lavoisier-Paris (1984). 